\title{
Commuting Quasihomogeneous Toeplitz Operator and Hankel Operator on Weighted Bergman Space
}

\author{
Jun Yang \\ Department of Mathematics, Shanghai Maritime University, Shanghai 201306, China \\ Correspondence should be addressed to Jun Yang; yangjundlut@gmail.com
}

Received 26 January 2013; Accepted 1 July 2013

Academic Editor: Giovanni P. Galdi

Copyright (C) 2013 Jun Yang. This is an open access article distributed under the Creative Commons Attribution License, which permits unrestricted use, distribution, and reproduction in any medium, provided the original work is properly cited.

We characterize the commuting Toeplitz operator and Hankel operator with quasihomogeneous symbols. Also, we use it to show the necessary and sufficient conditions for commuting Toeplitz operator and Hankel operator with ordinary functions.

\section{Introduction}

Let $d A$ denote Lebesgue area measure on the unit disk $\mathbb{D}$, normalized so that the measure of $\mathbb{D}$ equals 1 . For $\alpha>-1$, we denote by $d A_{\alpha}$ the measure $d A_{\alpha}(z)=(\alpha+1)\left(1-|z|^{2}\right)^{\alpha} d A(z)$. For $1 \leq p<+\infty$, the space $L^{p}\left(\mathbb{D}, d A_{\alpha}\right)$ is a Banach space. The weighted Bergman space $A_{\alpha}^{2}(\mathbb{D})$ is the closed subspace of analytic functions in the Hilbert space $L^{2}\left(\mathbb{D}, d A_{\alpha}\right)$. For each $z \in$ $\mathbb{D}$, the application $L_{z}: A_{\alpha}^{2}(\mathbb{D}) \rightarrow \mathbb{C}$ is continuous and can be represented as $L_{z}(f)=f(z)=\left\langle f, K_{z}^{(\alpha)}\right\rangle_{\alpha}$, where

$$
\begin{array}{r}
K_{z}^{(\alpha)}(w)=\frac{1}{(1-w \bar{z})^{2+\alpha}}=\sum_{n=0}^{\infty} \frac{\Gamma(n+2+\alpha)}{n ! \Gamma(2+\alpha)}(w \bar{z})^{n} \\
z, w \in \mathbb{D} .
\end{array}
$$

This means that, if $P_{\alpha}$ is the orthogonal projection from $L^{2}\left(\mathbb{D}, d A_{\alpha}\right)$ onto $A_{\alpha}^{2}(\mathbb{D})$, then $P_{\alpha}$ can be defined by

$$
\left(P_{\alpha} f\right)(w)=\left\langle f, K_{w}^{(\alpha)}\right\rangle_{\alpha}=\int_{\mathbb{D}} f(z) \frac{1}{(1-\bar{w} z)^{2+\alpha}} d A_{\alpha}(z) .
$$

For a function $f \in L^{\infty}\left(\mathbb{D}, d A_{\alpha}(z)\right)$, we define the Toeplitz operator $T_{f}: A_{\alpha}^{2}(\mathbb{D}) \rightarrow A_{\alpha}^{2}(\mathbb{D})$ with symbol $f$ by

$$
T_{f}(h)=P_{\alpha}(f h) \text {. }
$$

It is well known that

$$
T_{f}(h)(z)=\int_{\mathbb{D}} f(w) h(w) \overline{K_{z}^{(\alpha)}(w)} d A_{\alpha}(w), \quad z \in \mathbb{D} .
$$

Let $U^{(\alpha)}: L^{2}\left(\mathbb{D}, d A_{\alpha}(z)\right) \rightarrow L^{2}\left(\mathbb{D}, d A_{\alpha}(z)\right)$ be the unitary operator defined by $U^{(\alpha)} f(z)=\widetilde{f(z)}=f(\bar{z})$, where $f$ belongs to $L^{2}\left(\mathbb{D}, d A_{\alpha}(z)\right)$. Let $g$ be in $L^{2}\left(\mathbb{D}, d A_{\alpha}(z)\right)$; we define a bounded linear operator $M_{g}$ on $L^{2}\left(\mathbb{D}, d A_{\alpha}(z)\right)$ as follows:

$$
M_{g}(f)=g f
$$

Then we can define the small Hankel operator as follows:

$$
H_{g}: A_{\alpha}^{2}(\mathbb{D}) \longrightarrow A_{\alpha}^{2}(\mathbb{D})
$$

as $H_{g}=P_{\alpha} U^{(\alpha)} M_{g}$.

The study of commuting Toeplitz operators on the Bergman and Hardy spaces over various domains and related operator algebras has a long lasting history. On the Hardy space of the unit disk, Brown and Halmos [1] first showed that two Toeplitz operators are commuting if and only if either both symbols of these operators are analytic, or both symbols of these operators are coanalytic or a nontrivial linear combination of the symbols of these operators is constant. On the Bergman space, the situation is more complicated. Axler and Čucković obtained the analogous result for Toeplitz operators with bounded harmonic symbols on the Bergman space of 
the unit disk [2]. The problem of characterizing commuting Toeplitz operators with arbitrary bounded symbols seems quite challenging and is not fully understood until now. In [3], Čucković and Rao used the Mellin transform to characterize all Toeplitz operators on $L_{a}^{2}$ which commute with $T_{e^{i p \theta} r^{m}}$ for $(m, p) \in N \times N$. Later in [4] Louhichi and Zakariasy gave a partial characterization of commuting Toeplitz operators on $L_{a}^{2}$ with quasihomogeneous symbols. Recently, Lu and Zhang $[5,6]$ characterized the commuting Toeplitz operators and Hankel operators with quasihomogeneous symbols. There are also many other important results [7-13]. Motivated by those works, we study commuting Toeplitz operator and Hankel operator on the weighted Bergman space. In this paper, we obtain the necessary and sufficient conditions for commuting Toeplitz operator and Hankel operator.

An operator that will arise in our study of Toeplitz operators is the Mellin transform, defined for any function $\varphi \in$ $L^{1}([0,1], r d r)$; from the formula, $\widehat{\varphi_{\alpha}}(z)$ is the Mellin transform as follows:

$$
\widehat{\varphi_{\alpha}}(z)=\int_{0}^{1} \varphi(r)\left(1-r^{2}\right)^{\alpha} r^{z-1} d r
$$

which is a bounded holomorphic function in the half plane $\{z: \operatorname{Re} z>2\}$.

Let $\varphi \in L^{1}\left(\mathbb{D}, d A_{\alpha}\right)$ be a radial function; that is, suppose that $\varphi(z)=\varphi(|z|), z \in \mathbb{D}$. In fact, if we define the function $\varphi_{r}$ on $[0,1]$ by $\varphi_{r}(s)=\varphi(s)$, then a direct calculation shows that

$$
\left\langle T_{\varphi}\left(z^{k}\right), z^{l}\right\rangle_{\alpha}= \begin{cases}0 & \text { for } k \neq l \\ 2(\alpha+1) \widehat{\varphi_{\alpha}}(2 k+2) & \text { for } k=l\end{cases}
$$

so that if $k \in \mathbb{N}$,

$$
\begin{aligned}
T_{\varphi}\left(z^{k}\right)= & 2(1+\alpha) \frac{\Gamma(k+2+\alpha)}{k ! \Gamma(2+\alpha)} \\
& \times \int_{0}^{1} \varphi(r) r^{2 k+1}\left(1-|r|^{2}\right)^{\alpha} d r z^{k} \\
= & 2(1+\alpha) \frac{\Gamma(k+2+\alpha)}{k ! \Gamma(2+\alpha)} \widehat{\varphi_{\alpha}}(2 k+2) z^{k} .
\end{aligned}
$$

Thus, $T_{\varphi}$ is a diagonal operator on $A_{\alpha}^{2}(\mathbb{D})$ with coefficient sequence as follows:

$$
\left(2(1+\alpha) \frac{\Gamma(k+2+\alpha)}{k ! \Gamma(2+\alpha)} \widehat{\varphi_{\alpha}}(2 k+2)\right)_{k=0}^{\infty} .
$$

This makes it relatively simple to work with the product of two operators with such radial symbols.

Now, we define the "radialization" of a function $f \in L^{1}(\mathbb{D}$, $d A_{\alpha}$ ) by the following:

$$
\operatorname{rad}(f)(z)=\frac{1}{2 \pi} \int_{0}^{2 \pi} f\left(e^{i t} z\right) d t
$$

It is clear that a function $f$ is a radial if and only if $\operatorname{rad}(f)=f$.

Let $\Re^{\alpha}$ be the space of weighted square integrable radial functions on $\mathbb{D}$. By using that, trigonometric polynomials are dense in $L^{2}\left(\mathbb{D}, d A_{\alpha}\right)$ and that, for $k_{1} \neq k_{2}, e^{i k_{1} \theta} \Re^{\alpha}$ is orthogonal to $e^{i k_{2} \theta} \mathfrak{R}^{\alpha}$, we see that is

$$
L^{2}\left(\mathbb{D}, d A_{\alpha}\right)=\oplus_{k \in \mathbb{Z}} e^{i k \theta} \Re^{\alpha} .
$$

Definition 1. Let $\varphi$ be a function in $L^{1}\left(\mathbb{D}, d A_{\alpha}\right)$ which is of the form $e^{i k \theta} f$, where $f$ is a radial function. Then one says that $\varphi$ is a quasihomogeneous function of quasihomogeneous degree $k$.

A direct calculation gives the following lemmas which we will use often.

Lemma 2. Let $p \in \mathbb{N}$ and let $\varphi$ be an integrable radial function. Then,

$$
\begin{gathered}
T_{e^{i p \theta} \varphi}\left(z^{k}\right)=2(\alpha+1) \frac{\Gamma(k+p+2+\alpha)}{(k+p) ! \Gamma(2+\alpha)} \\
\quad \times \widehat{\varphi_{\alpha}}(2 k+p+2) z^{k+p}, \quad k \geq 0, \\
T_{e^{-i p \theta} \varphi}\left(z^{k}\right)=\left\{\begin{array}{cl}
0 & \text { if } 0 \leq k<p, \\
2(\alpha+1) \frac{\Gamma(k-p+2+\alpha)}{(k-p) ! \Gamma(2+\alpha)} & \\
\times \widehat{\varphi_{\alpha}}(2 k-p+2) z^{k-p} & \text { if } k \geq p .
\end{array}\right.
\end{gathered}
$$

Lemma 3. Let $\varphi$ be an integrable radial function. Then, for $p \in$ $\mathbb{Z}_{+}$,

$$
H_{e^{i p \theta} \varphi}\left(z^{k}\right)=0, \quad k \geq 0,
$$

and for $p \in \mathbb{N}$,

$H_{e^{-i p \theta} \varphi}\left(z^{k}\right)= \begin{cases}0 & \text { if } k>p, \\ 2(\alpha+1) \frac{\Gamma(-k+p+\alpha+2)}{(-k+p) ! \Gamma(2+\alpha)} & \\ \times \widehat{\varphi_{\alpha}}(p+2) z^{-k+p} & \text { if } 0 \leq k \leq p .\end{cases}$

\section{Commuting of Toeplitz Operator and Hankel Operator}

Theorem 4. Let $e^{i p \theta} f$ be a bounded function of quasihomogeneous degree $p \geq 0$ and $g=\sum_{k \in Z} e^{i k \theta} g_{k, \alpha}(r) \in L^{\infty}\left(\mathbb{D}, d A_{\alpha}\right)$. Then $T_{e^{i p \theta} f} H_{g}=H_{g} T_{e^{i p \theta} f}$ if and only if the following conditions holds

(1) $\widehat{f}_{\alpha}(2 j+p+2) \widehat{g}_{-j-k-p, \alpha}(k+j+p+2)=0$, if $0 \leq k \leq p-1$ and $j \geq 0$;

(2) $(\Gamma(k+2+\alpha) / k !) \widehat{f}_{\alpha}(2 k+p+2) \widehat{g}_{-j-k, \alpha}(k+j+2)=(\Gamma(j+$ $p+\alpha+2) /(j+p) !) \widehat{f}_{\alpha}(2 j+p+2) \widehat{g}_{-j-k-2 p, \alpha}(k+j+2 p+2)$, if $k \geq 0$ and $j \geq 0$. 
Proof. For $j \geq 0$,

$$
\begin{aligned}
& T_{e^{i p \theta} f} H_{g}\left(z^{j}\right)=T_{e^{i p \theta} f} H_{\sum_{k \in Z} e^{i k \theta} g_{k, \alpha}(r)}\left(z^{j}\right) \\
& =T_{e^{i p \theta} f}\left(\sum_{k=-j}^{-\infty} 2(\alpha+1) \frac{\Gamma(-j-k+\alpha+2)}{(-j-k) ! \Gamma(2+\alpha)}\right. \\
& \left.\times \widehat{g}_{k, \alpha}(-k+2) z^{-j-k}\right) \\
& =2 \sum_{k=-j}^{-\infty}(\alpha+1) \frac{\Gamma(-j-k+\alpha+2)}{(-j-k) ! \Gamma(2+\alpha)} \\
& \times \widehat{g}_{k, \alpha}(-k+2) T_{e^{i p \theta} f}\left(z^{-j-k}\right) \\
& =2 \sum_{k=-j}^{-\infty}(\alpha+1) \frac{\Gamma(-j-k+\alpha+2)}{(-j-k) ! \Gamma(2+\alpha)} \\
& \times \widehat{g}_{k, \alpha}(-k+2) 2(\alpha+1) \\
& \frac{\Gamma(-j-k+p+\alpha+2)}{(-j-k+p) ! \Gamma(2+\alpha)} \\
& \times \widehat{f}_{\alpha}(-2 j-2 k+p+2) z^{-j-k+p} \\
& =4(\alpha+1)^{2} \sum_{k=0}^{+\infty} \frac{\Gamma(k+\alpha+2)}{k ! \Gamma(2+\alpha)} \\
& \times \frac{\Gamma(k+p+\alpha+2)}{(k+p) ! \Gamma(2+\alpha)} \\
& \times \widehat{f}_{\alpha}(2 k+p+2) \\
& \cdot \widehat{g}_{-j-k, \alpha}(j+k+2) z^{k+p} \text {, } \\
& H_{g} T_{e^{i p \theta} f}\left(z^{j}\right)=H_{\sum_{k \in Z} e^{i k \theta} g_{k, \alpha}(r)} T_{e^{i p \theta} f}\left(z^{j}\right) \\
& =2(\alpha+1) \frac{\Gamma(j+p+\alpha+2)}{(j+p) ! \Gamma(2+\alpha)} \widehat{f}_{\alpha}(2 j+p+2) \\
& \times H_{\sum_{k \in Z} e^{i k \theta} g_{k, \alpha}(r)}\left(z^{j+p}\right) \\
& =2(\alpha+1) \frac{\Gamma(j+p+\alpha+2)}{(j+p) ! \Gamma(2+\alpha)} \widehat{f}_{\alpha}(2 j+p+2) \\
& \times \sum_{k=-j-p}^{-\infty} 2(\alpha+1) \\
& \cdot \frac{\Gamma(-j-k-p+\alpha+2)}{(-j-p-k) ! \Gamma(2+\alpha)} \widehat{g}_{k, \alpha}(-k+2) z^{-j-p-k} \\
& =\sum_{k=-j-p}^{-\infty} 4(\alpha+1)^{2} \frac{\Gamma(j+p+\alpha+2)}{(j+p) ! \Gamma(2+\alpha)} \\
& \times \frac{\Gamma(-j-k-p+\alpha+2)}{(-j-p-k) ! \Gamma(2+\alpha)}
\end{aligned}
$$

$$
\begin{aligned}
& \cdot \widehat{f}_{\alpha}(2 j+p+2) \widehat{g}_{k, \alpha}(-k+2) z^{-j-p-k} \\
& =4(\alpha+1)^{2} \sum_{k=0}^{+\infty} \frac{\Gamma(j+p+\alpha+2)}{(j+p) ! \Gamma(2+\alpha)} \\
& \times \frac{\Gamma(k+\alpha+2)}{(k) ! \Gamma(2+\alpha)} \widehat{f}_{\alpha}(2 j+p+2) \\
& \cdot \widehat{g}_{-j-p-k, \alpha}(k+j+p+2) z^{k} .
\end{aligned}
$$

If $T_{e^{i p \theta} f} H_{g}=H_{g} T_{e^{i p \theta} f}$, then we have

$$
\begin{aligned}
& \sum_{k=0}^{+\infty} \frac{\Gamma(k+\alpha+2)}{k ! \Gamma(2+\alpha)} \frac{\Gamma(k+p+\alpha+2)}{(k+p) ! \Gamma(2+\alpha)} \\
& \quad \times \widehat{f}_{\alpha}(2 k+p+2) \widehat{g}_{-j-k, \alpha}(j+k+2) z^{k+p} \\
& =\sum_{k=0}^{+\infty} \frac{\Gamma(j+p+\alpha+2)}{(j+p) ! \Gamma(2+\alpha)} \frac{\Gamma(k+\alpha+2)}{(k) ! \Gamma(2+\alpha)} \\
& \quad \times \widehat{f}_{\alpha}(2 j+p+2) \widehat{g}_{-j-p-k, \alpha}(k+j+p+2) z^{k},
\end{aligned}
$$

which is equivalent to

$$
\begin{aligned}
& \sum_{k=0}^{+\infty} \frac{\Gamma(k+\alpha+2)}{k ! \Gamma(2+\alpha)} \frac{\Gamma(k+p+\alpha+2)}{(k+p) ! \Gamma(2+\alpha)} \\
& \times \widehat{f}_{\alpha}(2 k+p+2) \widehat{g}_{-j-k, \alpha}(j+k+2) z^{k+p} \\
& =\sum_{k=0}^{p-1} \frac{\Gamma(j+p+\alpha+2)}{(j+p) ! \Gamma(2+\alpha)} \frac{\Gamma(k+\alpha+2)}{(k) ! \Gamma(2+\alpha)} \\
& \quad \times \widehat{f}_{\alpha}(2 j+p+2) \widehat{g}_{-j-p-k, \alpha} \\
& \quad \times(k+j+p+2) z^{k} \\
& +\sum_{k=p}^{+\infty} \frac{\Gamma(j+p+\alpha+2)}{(j+p) ! \Gamma(2+\alpha)} \frac{\Gamma(k+\alpha+2)}{(k) ! \Gamma(2+\alpha)} \\
& \quad \times \widehat{f}_{\alpha}(2 j+p+2) \widehat{g}_{-j-p-k, \alpha} \\
& \quad \times(k+j+p+2) z^{k}
\end{aligned}
$$

that is,

$$
\begin{aligned}
& \sum_{k=0}^{+\infty} \frac{\Gamma(k+\alpha+2)}{k ! \Gamma(2+\alpha)} \frac{\Gamma(k+p+\alpha+2)}{(k+p) ! \Gamma(2+\alpha)} \\
& \quad \times \widehat{f}_{\alpha}(2 k+p+2) \widehat{g}_{-j-k, \alpha} \\
& \quad \times(j+k+2) z^{k+p} \\
& =\sum_{k=0}^{p-1} \frac{\Gamma(j+p+\alpha+2)}{(j+p) ! \Gamma(2+\alpha)} \frac{\Gamma(k+\alpha+2)}{(k) ! \Gamma(2+\alpha)} \\
& \quad \times \widehat{f}_{\alpha}(2 j+p+2) \widehat{g}_{-j-p-k, \alpha}
\end{aligned}
$$




$$
\begin{aligned}
& \quad \times(k+j+p+2) z^{k} \\
& +\sum_{k=0}^{+\infty} \frac{\Gamma(j+p+\alpha+2)}{(j+p) ! \Gamma(2+\alpha)} \frac{\Gamma(p+k+\alpha+2)}{(p+k) ! \Gamma(2+\alpha)} \\
& \quad \times \widehat{f}_{\alpha}(2 j+p+2) \widehat{g}_{-j-2 p-k, \alpha} \\
& \quad \times(k+j+2 p+2) z^{p+k} .
\end{aligned}
$$

From the aforementioned we get the following.

Case 1. For $0 \leq k \leq p-1$ and $j \geq 0$,

$$
\widehat{f}_{\alpha}(2 j+p+2) \widehat{g}_{-j-p-k, \alpha}(k+j+p+2)=0 .
$$

Case 2. For $k \geq 0$ and $j \geq 0$,

$$
\begin{aligned}
\frac{\Gamma(k+\alpha+2)}{k !} \widehat{f}_{\alpha}(2 k+p+2) \widehat{g}_{-j-k, \alpha}(j+k+2) \\
=\frac{\Gamma(j+p+\alpha+2)}{(j+p) !} \widehat{f}_{\alpha}(2 j+p+2) \widehat{g}_{-j-2 p-k, \alpha} \\
\quad \times(k+j+2 p+2) .
\end{aligned}
$$

As a special case of Theorem 4, we can have the following corollary.

Corollary 5. Let $f$ be a bounded radial function and $g=$ $\sum_{k \in Z} e^{i k \theta} g_{k, \alpha}(r) \in L^{\infty}\left(\mathbb{D}, d A_{\alpha}\right)$. Then $T_{f} H_{g}=H_{g} T_{f}$ if and only if

$$
\begin{aligned}
& \frac{\Gamma(k+2+\alpha)}{k !} \widehat{f}_{\alpha}(2 k+2) \widehat{g}_{-j-k, \alpha}(k+j+2) \\
& \quad=\frac{\Gamma(j+\alpha+2)}{j !} \widehat{f}_{\alpha}(2 j+2) \widehat{g}_{-j-k, \alpha}(k+j+2),
\end{aligned}
$$

for $k \geq 0$ and $j \geq 0$.

Theorem 6. Let $e^{-i p \theta} f$ be a bounded function of quasihomogeneous degree $-p<0$ and $g=\sum_{k \in Z} e^{i k \theta} g_{k, \alpha}(r) \epsilon$ $L^{\infty}\left(\mathbb{D}, d A_{\alpha}\right)$. Then $T_{e^{-i p \theta} f} H_{g}=H_{g} T_{e^{-i p \theta} f}$ if and only if the following conditions holds

(1) $\widehat{f}_{\alpha}(2 k+p+2) \widehat{g}_{-j-k-p, \alpha}(k+j+p+2)=0$, if $k \geq 0$ and $p>j \geq 0$;

(2) $(\Gamma(k+p+2+\alpha) /(k+p) !) \widehat{f}_{\alpha}(2 k+p+2) \widehat{g}_{-j-k-p, \alpha}(k+$ $j+p+2)=(\Gamma(j-p+\alpha+2) /(j-p) !) \widehat{f}_{\alpha}(2 j-p+$ 2) $\widehat{g}_{-j-k+p, \alpha}(k+j-p+2)$, if $k \geq 0$ and $j \geq p$.
Proof. For $j \geq 0$, we have

$$
\begin{aligned}
& T_{e^{-i p \theta} f} H_{g}\left(z^{j}\right)=T_{e^{-i p \theta} f} H_{\sum_{k \in Z} e^{i k \theta} g_{k, \alpha}(r)}\left(z^{j}\right) \\
& =T_{e^{-i p \theta}} f^{2} \sum_{k=-j}^{-\infty}(\alpha+1) \frac{\Gamma(-j-k+\alpha+2)}{(-j-k) ! \Gamma(2+\alpha)} \\
& \times \widehat{\mathfrak{g}}_{k, \alpha}(-k+2)\left(z^{-j-k}\right) \\
& =2 \sum_{k=-j}^{-\infty}(\alpha+1) \frac{\Gamma(-j-k+\alpha+2)}{(-j-k) ! \Gamma(2+\alpha)} \\
& \times \widehat{g}_{k, \alpha}(-k+2) T_{e^{-i p \theta} f}\left(z^{-j-k}\right) \\
& =4(\alpha+1)^{2} \sum_{k=-j-p}^{-\infty} \frac{\Gamma(-j-k+\alpha+2)}{(-j-k) ! \Gamma(2+\alpha)} \\
& \times \widehat{g}_{k, \alpha}(-k+2) \\
& \frac{\Gamma(-j-k-p+\alpha+2)}{(-j-k-p) ! \Gamma(2+\alpha)} \\
& \times \widehat{f}_{\alpha}(-2 j-2 k-p+2) z^{-j-k-p} \\
& =4(\alpha+1)^{2} \sum_{k=0}^{+\infty} \frac{\Gamma(k+p+\alpha+2)}{(k+p) ! \Gamma(2+\alpha)} \\
& \times \widehat{g}_{-k-j-p, \alpha}(k+j+p+2) \\
& \frac{\Gamma(k+\alpha+2)}{k ! \Gamma(2+\alpha)} \widehat{f}_{\alpha}(2 k+p+2) z^{k} \text {. }
\end{aligned}
$$

Then one has the following.

Case 1. For $p>j \geq 0$,

$$
H_{g} T_{e^{-i p \theta} f}\left(z^{j}\right)=H_{\sum_{k \in Z} e^{i k \theta} g_{k, \alpha}(r)} T_{e^{-i p \theta} f}\left(z^{j}\right)=0 .
$$

Case 2. For $j \geq p$,

$$
\begin{aligned}
H_{g} T_{e^{-i p \theta} f}\left(z^{j}\right)= & H_{\sum_{k \in Z} e^{i k \theta} g_{k, \alpha}(r)} T_{e^{-i p \theta} f}\left(z^{j}\right) \\
= & 2(\alpha+1) \frac{\Gamma(j-p+\alpha+2)}{(j-p) ! \Gamma(2+\alpha)} \\
& \times \widehat{f}_{\alpha}(2 j-p+2) H_{\sum_{k \in Z} e^{i k \theta} g_{k, \alpha}(r)}\left(z^{j-p}\right) \\
= & 4(\alpha+1)^{2} \sum_{k=p-j}^{-\infty} \frac{\Gamma(j-p+\alpha+2)}{(j-p) ! \Gamma(2+\alpha)} \\
\times \widehat{f}_{\alpha}(2 j-p+2) & \times \frac{\Gamma(-j+p-k+\alpha+2)}{(-j+p-k) ! \Gamma(2+\alpha)} \\
& \cdot \widehat{g}_{k, \alpha}(-k+2) z^{-j+p-k}
\end{aligned}
$$




$$
\begin{aligned}
=4(\alpha+1)^{2} \sum_{k=0}^{+\infty} & \frac{\Gamma(j-p+\alpha+2)}{(j-p) ! \Gamma(2+\alpha)} \\
& \times \widehat{f}_{\alpha}(2 j-p+2) \\
& \times \frac{\Gamma(k+\alpha+2)}{k ! \Gamma(2+\alpha)} \\
& \times \widehat{g}_{-j-k+p, \alpha}(j+k-p+2) z^{k} .
\end{aligned}
$$

If $T_{e^{-i p \theta} f} H_{g}=H_{g} T_{e^{-i p \theta} f}$, then we have the following.

Case 1 . For $p>j \geq 0$,

$$
\begin{aligned}
& \sum_{k=0}^{+\infty} \frac{\Gamma(k+p+\alpha+2)}{(k+p) ! \Gamma(2+\alpha)} \widehat{g}_{-k-j-p, \alpha}(k+j+p+2) \\
& \quad \times \frac{\Gamma(k+\alpha+2)}{k ! \Gamma(2+\alpha)} \widehat{f}_{\alpha}(2 k+p+2) z^{k}=0 .
\end{aligned}
$$

Case 2. For $j \geq p$,

$$
\begin{gathered}
\sum_{k=0}^{+\infty} \frac{\Gamma(k+p+\alpha+2)}{(k+p) ! \Gamma(2+\alpha)} \widehat{g}_{-k-j-p, \alpha}(k+j+p+2) \\
\times \frac{\Gamma(k+\alpha+2)}{k ! \Gamma(2+\alpha)} \widehat{f}_{\alpha}(2 k+p+2) z^{k} \\
=\sum_{k=0}^{+\infty} \frac{\Gamma(j-p+\alpha+2)}{(j-p) ! \Gamma(2+\alpha)} \widehat{f}_{\alpha}(2 j-p+2) \\
\times \frac{\Gamma(k+\alpha+2)}{k ! \Gamma(2+\alpha)} \widehat{g}_{-j-k+p, \alpha} \\
\times(j+k-p+2) z^{k} .
\end{gathered}
$$

That is one has the following.

Case 1. For $p>j \geq 0, k \geq 0$,

$$
\widehat{g}_{-k-j-p, \alpha}(k+j+p+2) \widehat{f}_{\alpha}(2 k+p+2)=0 .
$$

Case 2. For $j \geq p, k \geq 0$,

$$
\begin{aligned}
\frac{\Gamma(k+p+\alpha+2)}{(k+p) !} \widehat{g}_{-k-j-p, \alpha} \\
\quad \times(k+j+p+2) \widehat{f}_{\alpha}(2 k+p+2) \\
=\frac{\Gamma(j-p+\alpha+2)}{(j-p) !} \widehat{f}_{\alpha}(2 j-p+2) \\
\quad \times \widehat{g}_{-j-k+p, \alpha}(j+k-p+2) .
\end{aligned}
$$

Theorem 7. Let $e^{-i s \theta} g$ be a bounded function of quasihomogeneous degree $-s \leq 0$ and $f=\sum_{k \in Z} e^{i k \theta} f_{k, \alpha}(r) \in L^{\infty}\left(\mathbb{D}, d A_{\alpha}\right)$. Then $T_{f} H_{e^{-i s \theta} g}=H_{e^{-i s \theta} g} T_{f}$ if and only if $\widehat{g}_{\alpha}(s+2)=0$ or

(1) $\widehat{f}_{k-j, \alpha}(k+j+2)=0$, if $s \geq k \geq 0$ and $j>s$;
(2) $(\Gamma(-j+s+\alpha+2) /(-j+s) !) \widehat{f}_{k+j-s, \alpha}(-j+s+k+2)=$ $(\Gamma(s-k+\alpha+2) /(s-k) !) \widehat{f}_{s-k-j, \alpha}(s-k+j+2)$, if $s \geq k \geq 0$ and $s \geq j \geq 0$;

(3) $\widehat{f}_{k+j-s, \alpha}(-j+s+k+2)=0$, if $k>s$ and $s \geq j \geq 0$.

Proof. For $j \geq 0$, we have the following.

Case 1. For $j>s$,

$$
T_{f} H_{e^{-i s \theta} g}\left(z^{j}\right)=T_{\sum_{k \in Z} e^{i k \theta} f_{k, \alpha}(r)} H_{e^{-i s \theta} g}\left(z^{j}\right)=0 .
$$

Case 2. For $s \geq j \geq 0$,

$$
\begin{aligned}
& T_{f} H_{e^{-i s \theta} g}\left(z^{j}\right) \\
& =T_{\sum_{k \in Z} e^{i k \theta} f_{k, \alpha}(r)} H_{e^{-i s \theta} g}\left(z^{j}\right) \\
& =2(\alpha+1) \frac{\Gamma(-j+s+\alpha+2)}{(-j+s) ! \Gamma(2+\alpha)} \widehat{g}_{\alpha}(s+2) \\
& \times T_{\sum_{k \in Z} e^{i k \theta} f_{k, \alpha}(r)}\left(z^{-j+s}\right) \\
& =4(\alpha+1)^{2} \frac{\Gamma(-j+s+\alpha+2)}{(-j+s) ! \Gamma(2+\alpha)} \widehat{g}_{\alpha}(s+2) \\
& \times \sum_{k=j-s}^{+\infty} \frac{\Gamma(s-j+k+\alpha+2)}{(s-j+k) ! \Gamma(2+\alpha)} \widehat{f}_{k, \alpha} \\
& \times(2 s-2 j+k+2) z^{-j+s+k} \\
& H_{e^{-i s \theta}} T_{f}\left(z^{j}\right) \\
& =H_{e^{-i s \theta} g} T_{\sum_{k \in Z} e^{i k \theta} f_{k, \alpha}(r)}\left(z^{j}\right) \\
& =H_{e^{-i s \theta}} g\left(\sum_{k=-j}^{+\infty} 2(\alpha+1)\right. \\
& \times \frac{\Gamma(j+k+\alpha+2)}{(j+k) ! \Gamma(2+\alpha)} \\
& \left.\times \widehat{f}_{k, \alpha}(2 j+k+2) z^{j+k}\right) \\
& =2 \sum_{k=0}^{+\infty}(\alpha+1) \frac{\Gamma(k+\alpha+2)}{k ! \Gamma(2+\alpha)} \\
& \times \widehat{f}_{k-j, \alpha}(k+j+2) H_{e^{-i s \theta} g}\left(z^{k}\right) \\
& =4(\alpha+1)^{2} \sum_{k=0}^{s} \frac{\Gamma(k+\alpha+2)}{k ! \Gamma(2+\alpha)} \\
& \times \frac{\Gamma(-k+s+\alpha+2)}{(-k+s) ! \Gamma(2+\alpha)} \widehat{f}_{k-j, \alpha} \\
& \times(k+j+2) \widehat{g}_{\alpha}(s+2) z^{-k+s} \text {. }
\end{aligned}
$$


Applying $H_{e^{-i s \theta} g} T_{f}=T_{f} H_{e^{-i s \theta} g}$, we have the following.

Case 1. For $j>s$,

$$
\begin{aligned}
4(\alpha+1)^{2} \sum_{k=0}^{s} & \frac{\Gamma(k+\alpha+2)}{k ! \Gamma(2+\alpha)} \frac{\Gamma(-k+s+\alpha+2)}{(-k+s) ! \Gamma(2+\alpha)} \\
& \times \widehat{f}_{k-j, \alpha}(k+j+2) \widehat{g}_{\alpha}(s+2) z^{-k+s}=0 .
\end{aligned}
$$

Case 2. For $s \geq j \geq 0$,

$$
\begin{gathered}
\sum_{k=j-s}^{+\infty} \frac{\Gamma(s-j+k+\alpha+2)}{(s-j+k) ! \Gamma(2+\alpha)} \widehat{f}_{k, \alpha}(2 s-2 j+k+2) \\
\quad \times \frac{\Gamma(-j+s+\alpha+2)}{(-j+s) ! \Gamma(2+\alpha)} \widehat{g}_{\alpha}(s+2) z^{-j+s+k} \\
=\sum_{k=0}^{s} \frac{\Gamma(k+\alpha+2)}{k ! \Gamma(2+\alpha)} \frac{\Gamma(-k+s+\alpha+2)}{(-k+s) ! \Gamma(2+\alpha)} \\
\quad \times \widehat{f}_{k-j, \alpha}(k+j+2) \widehat{g}_{\alpha}(s+2) z^{-k+s} .
\end{gathered}
$$

If $\widehat{g}_{\alpha}(s+2)=0$, then the equation holds.

Otherwise $\widehat{g}_{\alpha}(s+2) \neq 0$, we have the following.

Case 1. For $j>s$,

$$
\begin{gathered}
\sum_{k=0}^{s} \frac{\Gamma(k+\alpha+2)}{k ! \Gamma(2+\alpha)} \frac{\Gamma(-k+s+\alpha+2)}{(-k+s) ! \Gamma(2+\alpha)} \\
\times \widehat{f}_{k-j, \alpha}(k+j+2) z^{-k+s}=0 .
\end{gathered}
$$

Case 2. For $s \geq j \geq 0$,

$$
\begin{aligned}
& \sum_{k=j-s}^{+\infty} \frac{\Gamma(s-j+k+\alpha+2)}{(s-j+k) ! \Gamma(2+\alpha)} \widehat{f}_{k, \alpha}(2 s-2 j+k+2) \\
& \quad \times \frac{\Gamma(-j+s+\alpha+2)}{(-j+s) ! \Gamma(2+\alpha)} z^{-j+s+k} \\
& =\sum_{k=0}^{s} \frac{\Gamma(k+\alpha+2)}{k ! \Gamma(2+\alpha)} \frac{\Gamma(-k+s+\alpha+2)}{(-k+s) ! \Gamma(2+\alpha)} \\
& \quad \times \widehat{f}_{k-j, \alpha}(k+j+2) z^{-k+s} .
\end{aligned}
$$

that is one has the following.

Case 1. For $s \geq k \geq 0, j>s$,

$$
\widehat{f}_{k-j, \alpha}(k+j+2) z^{-k+s}=0 \text {. }
$$

Case 2. For $s \geq j \geq 0$,

$$
\begin{aligned}
& \sum_{k=0}^{+\infty} \frac{\Gamma(s-j+\alpha+2)}{(s-j) ! \Gamma(2+\alpha)} \widehat{f}_{k+j-s, \alpha} \\
& \quad \times(s-j+k+2) \frac{\Gamma(k+\alpha+2)}{k ! \Gamma(2+\alpha)} z^{k} \\
& =\sum_{k=0}^{s} \frac{\Gamma(k+\alpha+2)}{k ! \Gamma(2+\alpha)} \frac{\Gamma(-k+s+\alpha+2)}{(-k+s) ! \Gamma(2+\alpha)} \\
& \quad \times \widehat{f}_{s-k-j, \alpha}(s-k+j+2) z^{k} .
\end{aligned}
$$

Then we get the following.

Case 1. For $s \geq k \geq 0, j>s, \widehat{f}_{k-j, \alpha}(k+j+2)=0$.

Case 2. For $s \geq j \geq 0$,

$$
\begin{aligned}
& \frac{\Gamma(s-j+\alpha+2)}{(s-j) !} \widehat{f}_{k+j-s, \alpha}(s-j+k+2) \\
& \quad=\frac{\Gamma(-k+s+\alpha+2)}{(-k+s) !} \widehat{f}_{s-k-j, \alpha}(s-k+j+2) .
\end{aligned}
$$

Case 3. For $k>s, s \geq j \geq 0, \widehat{f}_{k+j-s, \alpha}(-j+s+k+2)=0$.

Corollary 8. Let $g$ be a bounded radial function and $f=$ $\sum_{k \in Z} e^{i k \theta} f_{k, \alpha}(r) \in L^{\infty}\left(\mathbb{D}, d A_{\alpha}\right)$. Then $T_{f} H_{g}=H_{g} T_{f}$ if and only if

(1) $\widehat{g}_{\alpha}(s+2)=0$;

(2) $\widehat{f}_{k, \alpha}(k+2)=0$ and $\widehat{f}_{-k, \alpha}(k+2)=0$, for $k>0$.

Finally, we will investigate the situation that both functions are ordinary functions.

Theorem 9. Let $f=\sum_{k \in Z} e^{i k \theta} f_{k, \alpha}(r) \in L^{\infty}\left(\mathbb{D}, d A_{\alpha}\right)$ and $g=$ $\sum_{\text {if }}{ }_{l \in Z} e^{i l \theta} g_{l, \alpha}(r) \in L^{\infty}\left(\mathbb{D}, d A_{\alpha}\right)$. Then $T_{f} H_{g}=H_{g} T_{f}$ if and only

$$
\begin{aligned}
& \sum_{k=0}^{+\infty} \frac{\Gamma(k+\alpha+2)}{k ! \Gamma(2+\alpha)} \widehat{f}_{k-m, \alpha}(m+k+2) \\
& \times \widehat{g}_{-n-k, \alpha}(n+k+2) \\
& =\sum_{k=0}^{+\infty} \frac{\Gamma(k+\alpha+2)}{k ! \Gamma(2+\alpha)} \widehat{f}_{n-k, \alpha}(n+k+2) \\
& \quad \times \widehat{g}_{-k-m, \alpha}(k+m+2),
\end{aligned}
$$

for $m \geq 0$ and $n \geq 0$. 
Proof. For $j \geq 0$, we have

$$
\begin{aligned}
& H_{g} T_{f}\left(z^{j}\right)=H_{\sum_{l \in Z} e^{i l \theta} g_{l, \alpha}(r)} T_{\sum_{k \in Z} e^{i k \theta} f_{k, \alpha}(r)}\left(z^{j}\right) \\
& =H_{\sum_{l \in Z} e^{i l \theta} g_{l, \alpha}(r)}\left(\sum_{k=-j}^{+\infty} 2(\alpha+1)\right. \\
& \times \frac{\Gamma(j+k+\alpha+2)}{(j+k) ! \Gamma(2+\alpha)} \\
& \left.\times \widehat{f}_{k, \alpha}(2 j+k+2) z^{j+k}\right) \\
& =\sum_{k=-j}^{+\infty} 2(\alpha+1) \frac{\Gamma(j+k+\alpha+2)}{(j+k) ! \Gamma(2+\alpha)} \\
& \times \widehat{f}_{k, \alpha}(2 j+k+2) \\
& \left(\sum_{l=-(j+k)}^{-\infty} 2(\alpha+1) \frac{\Gamma(-j-k-l+\alpha+2)}{(-j-k-l) ! \Gamma(2+\alpha)}\right. \\
& \left.\times \widehat{g}_{l, \alpha}(-l+2) z^{-k-j-l}\right) \\
& =\sum_{k=0}^{+\infty} 2(\alpha+1) \frac{\Gamma(k+\alpha+2)}{k ! \Gamma(2+\alpha)} \\
& \times \widehat{f}_{k-j, \alpha}(j+k+2) \\
& \cdot\left(\sum_{l=0}^{+\infty} 2(\alpha+1) \frac{\Gamma(l+\alpha+2)}{l ! \Gamma(2+\alpha)}\right. \\
& \left.\times \widehat{g}_{-l-k, \alpha}(l+k+2) z^{l}\right)
\end{aligned}
$$$$
T_{f} H_{g}\left(z^{j}\right)=T_{\sum_{k \in Z} e^{i k \theta} f_{k, \alpha}(r)} H_{\sum_{l \in Z}} e^{i l \theta} g_{l, \alpha}(r)\left(z^{j}\right)
$$$$
=T_{\sum_{k \in Z} e^{i k \theta} f_{k, \alpha}(r)}\left(\sum_{l=-j}^{-\infty} 2(\alpha+1)\right.
$$$$
\times \frac{\Gamma(-j-l+\alpha+2)}{(-j-l) ! \Gamma(2+\alpha)}
$$$$
\left.\times \widehat{g}_{l, \alpha}(-l+2) z^{-j-l}\right)
$$$$
=\sum_{l=-j}^{-\infty} 2(\alpha+1) \frac{\Gamma(-j-l+\alpha+2)}{(-j-l) ! \Gamma(2+\alpha)}
$$$$
\times \widehat{g}_{l, \alpha}(-l+2)
$$$$
\cdot\left(\sum_{k=j+l}^{+\infty} 2(\alpha+1) \frac{\Gamma(-j-l+k+\alpha+2)}{(-j-l+k) ! \Gamma(2+\alpha)}\right.
$$

$$
\begin{aligned}
& \left.\times \widehat{f}_{k, \alpha}(-2 j-2 l+k+2) z^{-j-l+k}\right) \\
& =\sum_{k=0}^{+\infty} 2(\alpha+1) \frac{\Gamma(k+\alpha+2)}{k ! \Gamma(2+\alpha)} \\
& \quad \times \widehat{g}_{-k-j, \alpha}(k+j+2) \\
& \cdot\left(\sum_{l=0}^{+\infty} 2(\alpha+1) \frac{\Gamma(l+\alpha+2)}{l ! \Gamma(2+\alpha)}\right. \\
& \left.\quad \times \widehat{f}_{l-k, \alpha}(l+k+2) z^{l}\right) .
\end{aligned}
$$

From the aforementioned we get, for $m \geq 0$ and $n \geq 0$,

$$
\begin{aligned}
& \left(H_{g} T_{f}\left(z^{m}\right), z^{n}\right)=\left(\sum_{k=0}^{+\infty} 2(\alpha+1) \frac{\Gamma(k+\alpha+2)}{k ! \Gamma(2+\alpha)} \widehat{f}_{k-m, \alpha}\right. \\
& \times(m+k+2) \\
& \cdot \sum_{l=0}^{+\infty} 2(\alpha+1) \frac{\Gamma(l+\alpha+2)}{l ! \Gamma(2+\alpha)} \widehat{g}_{-l-k, \alpha} \\
& \left.\times(l+k+2) z^{l}, z^{n}\right) \\
& =\left(\sum_{k=0}^{+\infty} 2(\alpha+1) \frac{\Gamma(k+\alpha+2)}{k ! \Gamma(2+\alpha)}\right. \\
& \times \widehat{f}_{k-m, \alpha}(m+k+2) \\
& \cdot 2(\alpha+1) \frac{\Gamma(n+\alpha+2)}{n ! \Gamma(2+\alpha)} \widehat{g}_{-n-k, \alpha} \\
& \left.\times(n+k+2) z^{n}, z^{n}\right) \\
& =4(\alpha+1)^{2} \sum_{k=0}^{+\infty} \frac{\Gamma(k+\alpha+2)}{k ! \Gamma(2+\alpha)} \widehat{f}_{k-m, \alpha} \\
& \times(m+k+2) \widehat{g}_{-n-k, \alpha} \\
& \times(n+k+2), \\
& \left(T_{f} H_{g}\left(z^{m}\right), z^{n}\right)=\left(\sum_{k=0}^{+\infty} 2(\alpha+1) \frac{\Gamma(k+\alpha+2)}{k ! \Gamma(2+\alpha)}\right. \\
& \times \widehat{g}_{-k-m, \alpha}(k+m+2) \\
& \cdot \sum_{l=0}^{+\infty} 2(\alpha+1) \frac{\Gamma(l+\alpha+2)}{l ! \Gamma(2+\alpha)} \\
& \left.\times \widehat{f}_{l-k, \alpha}(l+k+2) z^{l}, z^{n}\right)
\end{aligned}
$$




$$
\begin{aligned}
& =\left(\sum_{k=0}^{+\infty} 2(\alpha+1) \frac{\Gamma(k+\alpha+2)}{k ! \Gamma(2+\alpha)}\right. \\
& \quad \times \widehat{g}_{-k-m, \alpha}(k+m+2) \\
& \cdot 2(\alpha+1) \frac{\Gamma(n+\alpha+2)}{n ! \Gamma(2+\alpha)} \widehat{f}_{n-k, \alpha} \\
& \left.\quad \times(n+k+2) z^{n}, z^{n}\right) \\
& =4(\alpha+1)^{2} \sum_{k=0}^{+\infty} \frac{\Gamma(k+\alpha+2)}{k ! \Gamma(2+\alpha)} \\
& \times \widehat{f}_{n-k, \alpha}(n+k+2) \\
& \times \widehat{g}_{-k-m, \alpha}(k+m+2) .
\end{aligned}
$$

If $H_{g} T_{f}=T_{f} H_{g}$, then we get

$$
\begin{aligned}
\sum_{k=0}^{+\infty} & \frac{\Gamma(k+\alpha+2)}{k ! \Gamma(2+\alpha)} \widehat{f}_{k-m, \alpha}(m+k+2) \\
& \times \widehat{g}_{-n-k, \alpha}(n+k+2) \\
= & \sum_{k=0}^{+\infty} \frac{\Gamma(k+\alpha+2)}{k ! \Gamma(2+\alpha)} \widehat{f}_{n-k, \alpha}(n+k+2) \\
& \times \widehat{g}_{-k-m, \alpha}(k+m+2),
\end{aligned}
$$

for $m \geq 0$ and $n \geq 0$.

The converse is easy to get.

\section{Acknowledgments}

The author would like to thank the referees for their valuable comments and suggestions, which helped to improve the paper. This work is supported by the National Natural Science Foundation of China (no. 11126061), Innovation Program of Shanghai Municipal Education Commission (no. 13YZ090) and the Science \& Technology Program of Shanghai Maritime University (no. 20120098).

\section{References}

[1] A. Brown and P. R. Halmos, "Algebraic properties of Toeplitz operators," Journal für die Reine und Angewandte Mathematik, vol. 213, pp. 89-102, 1963/1964.

[2] S. Axler and Ž. Čučković, "Commuting Toeplitz operators with harmonic symbols," Integral Equations and Operator Theory, vol. 14, no. 1, pp. 1-12, 1991.

[3] Ž. Čučković and N. V. Rao, "Mellin transform, monomial symbols, and commuting Toeplitz operators," Journal of Functional Analysis, vol. 154, no. 1, pp. 195-214, 1998.

[4] I. Louhichi and L. Zakariasy, "On Toeplitz operators with quasihomogeneous symbols," Archiv der Mathematik, vol. 85, no. 3, pp. 248-257, 2005.
[5] Y. F. Lu and B. Zhang, "Commuting Hankel and Toeplitz operators on the Bergman space," Chinese Annals of Mathematics A, vol. 32, no. 5, pp. 519-530, 2011.

[6] B. Zhang, Y. Shi, and Y. Lu, "Algebraic properties of Toeplitz operators on the polydisk," Abstract and Applied Analysis, vol. 2011, Article ID 962313, 18 pages, 2011.

[7] B. Zhang and Y. Lu, "Toeplitz operators with quasihomogeneous symbols on the Bergman space of the unit ball," Journal of Function Spaces and Applications, vol. 2012, Article ID 414201, 16 pages, 2012.

[8] X. Dong and Z. Zhou, "Commuting quasihomogeneous Toeplitz operators on the harmonic Bergman space," Complex Analysis and Operator Theory, 2012.

[9] B. R. Choe, H. Koo, and Y. J. Lee, "Commuting Toeplitz operators on the polydisk," Transactions of the American Mathematical Society, vol. 356, no. 5, pp. 1727-1749, 2004.

[10] Y. J. Lee, "Commuting Toeplitz operators on the Hardy space of the polydisk," Proceedings of the American Mathematical Society, vol. 138, no. 1, pp. 189-197, 2010.

[11] J. Yang, L. Liu, and Y. Lu, "Algebraic properties of Toeplitz operators on the pluriharmonic Bergman space," Journal of Function Spaces and Applications, vol. 2013, Article ID 578436, 12 pages, 2013.

[12] N. L. Vasilevski, "Bergman space structure, commutative algebras of Toeplitz operators, and hyperbolic geometry," Integral Equations and Operator Theory, vol. 46, no. 2, pp. 235-251, 2003.

[13] N. L. Vasilevski, Commutative Algebras of Toeplitz Operators on the Bergman Space, vol. 185 of Operator Theory: Advances and Applications, Birkhäuser, Basel, Switzerland, 2008. 


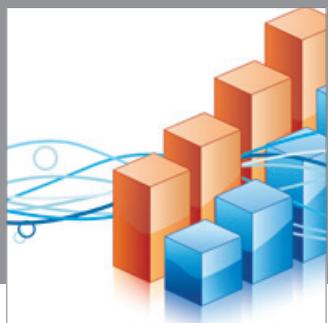

Advances in

Operations Research

mansans

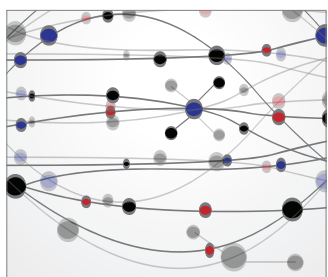

The Scientific World Journal
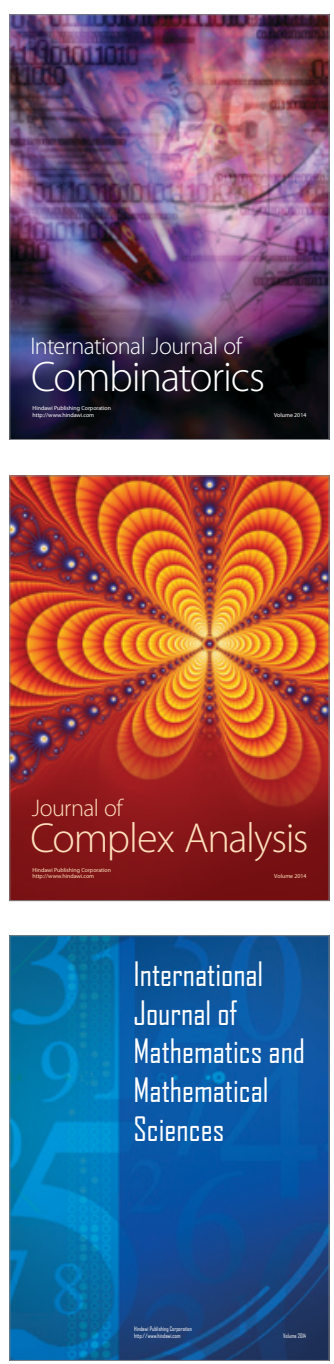
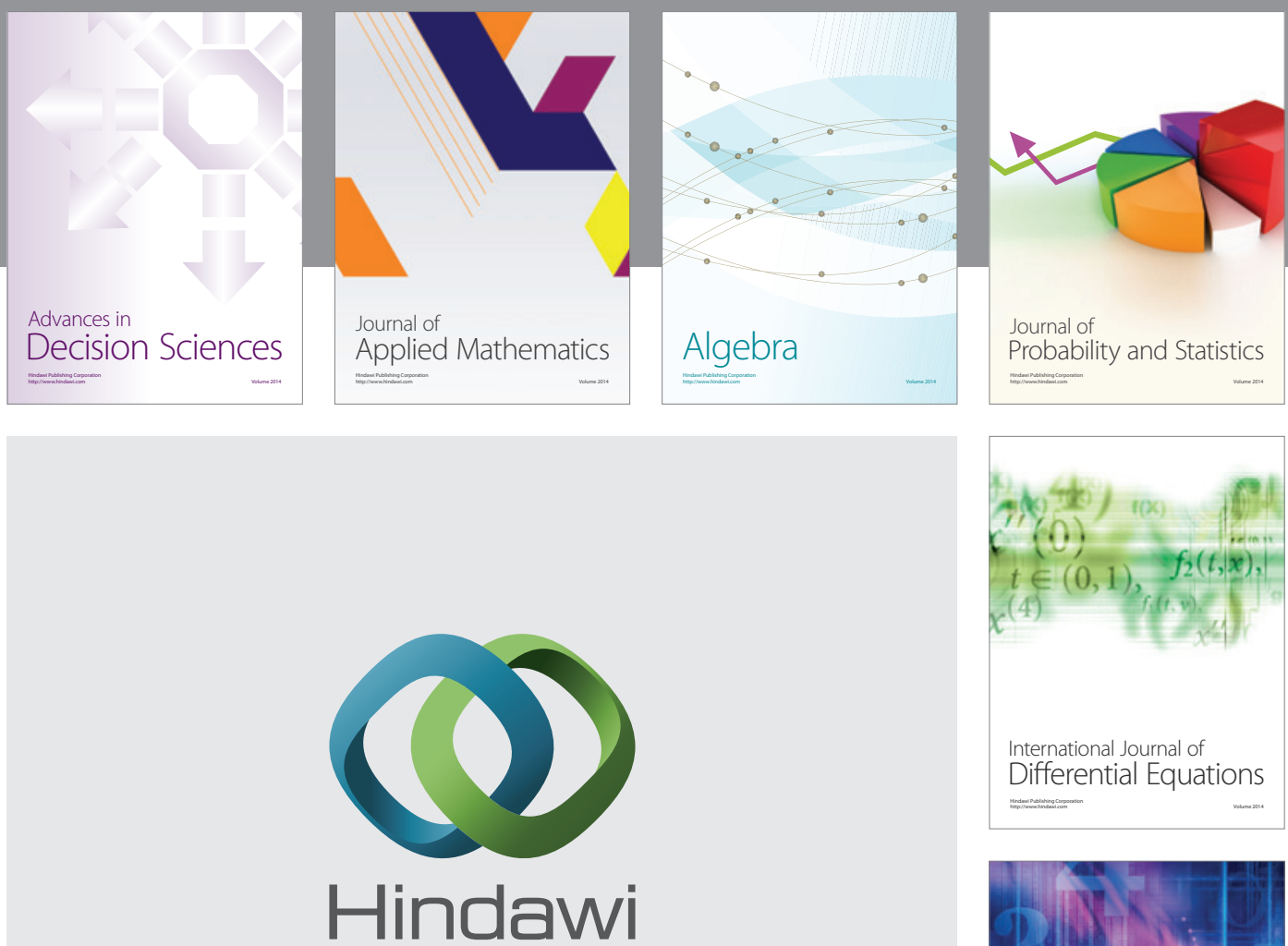

Submit your manuscripts at http://www.hindawi.com
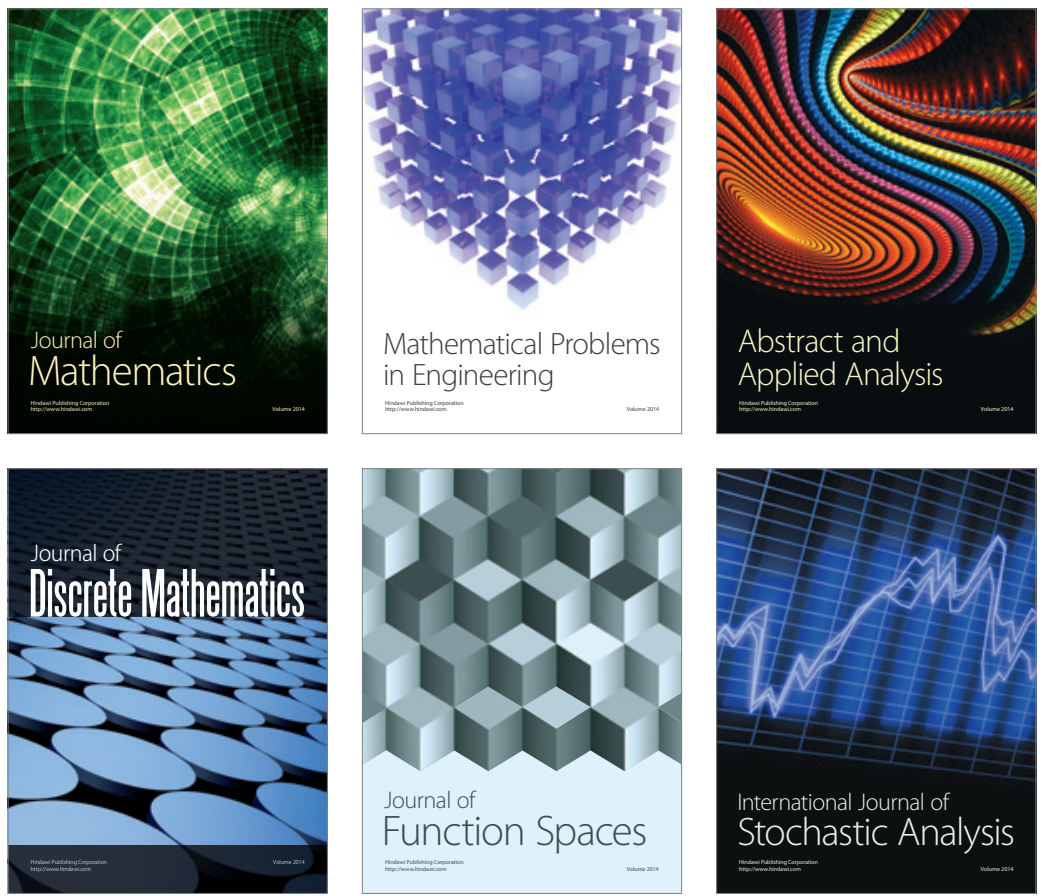

Journal of

Function Spaces

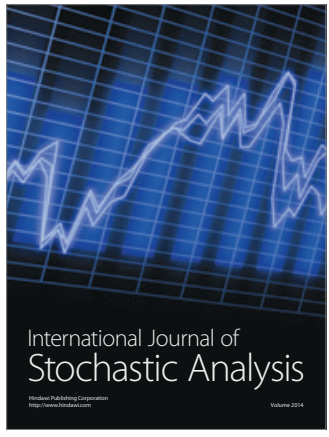

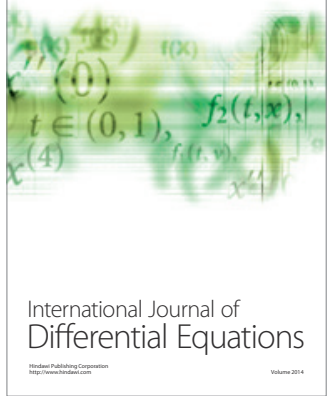
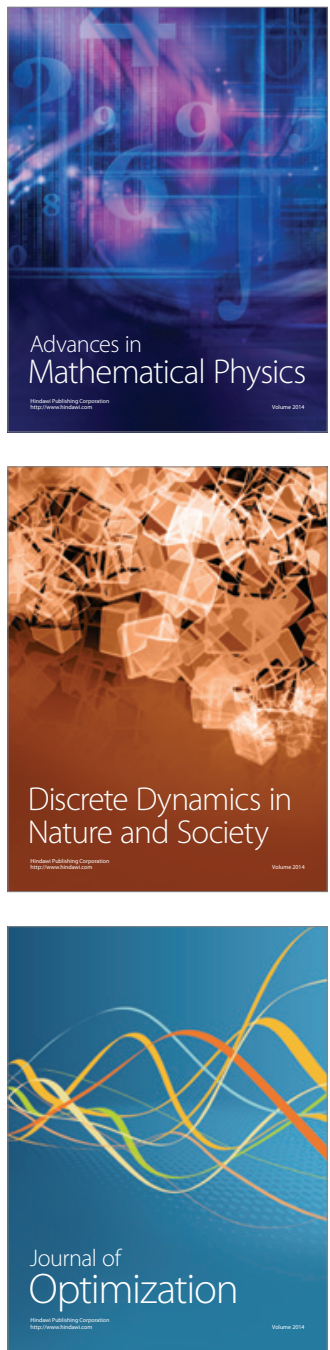